\title{
Single-day treatment for orolabial and genital herpes: a brief review of pathogenesis and pharmacology
}

\author{
Sapna Modi' \\ Livia Van' \\ Aron Gewirtzman ${ }^{2}$ \\ Natalia Mendoza ${ }^{2}$ \\ Brenda Bartlett ${ }^{2}$ \\ Anne Marie Tremaine ${ }^{2}$ \\ Stephen Tyring ${ }^{3}$ \\ 'Baylor College of Medicine, Houston, \\ TX, USA; ${ }^{2}$ Center for Clinical Studies, \\ Houston, TX, USA; ${ }^{3}$ Department of \\ Dermatology, University of Texas \\ Health Science Center, Houston, TX, \\ USA
}

Correspondence: Aron Gewirtzman Center for Clinical Studies, 6655 Travis, Suite I20, Houston, TX 77030, USA

$\mathrm{Tel}+|7| 35288818$

Fax + I 7135288848

Email agewirtzman@dermtexas.com

\begin{abstract}
Herpes simplex virus (HSV) infection is a highly prevalent condition responsible for significant morbidity and occasional mortality each year. Approximately half of all patients infected by HSV will experience at least one recurrence in their lifetime. For these recurrences, traditional therapy has included both suppressive and episodic treatment with nucleoside analogs. In regards to episodic treatment, 2- to 5-day oral regimens are best studied and most commonly reported. As with any medical condition having a well-understood mechanism of action and targeted treatment, therapeutic intervention is only as effective as allowed by patient compliance. Based on these concerns, recent studies have focused on shorter, less complicated, and more affordable options. This review delineates the evidence for single-day treatments of orolabial and genital herpes. Randomized, double-blind studies of both valacyclovir and famciclovir as single-day episodic therapy for HSV have been reported in the literature. Although no headto-head studies between the drugs have been performed, both regimens produced significant improvement in healing time and symptom resolution over placebo. Single-day therapy for HSV infection is appealing for multiple reasons. First, it simplifies the regimen, increasing likelihood of patient compliance. Additionally, it allows complete delivery of the medication at the onset of symptoms, when viral replication is highest and intervention has greatest effect. Lastly, the reduced number of pills necessary for single versus multiple day therapy decreases the overall cost of treatment per episode, an important factor in modern-day healthcare.
\end{abstract}

Keywords: famciclovir, genital herpes, orolabial herpes, patient-initiated episodic therapy, single-day, antiviral

\section{Introduction}

Herpes simplex virus (HSV) causes an incurable viral infection that affects over 40 million people in the United States, with over 600,000 cases diagnosed each year (Nadelman and Newcomer 2000). The virus spreads through close person-to-person contact, breeching the mucocutaneous barrier by direct mucosal penetration or through microabrasions in the skin. When previously unaffected individuals acquire a herpes infection, they develop neutralizing antibodies against HSV. Following this primary infection, the virus remains latent in the dorsal root ganglia until some trigger incites reactivation (Nadelman and Newcomer 2000). Dormancy gives these viruses the unique ability to cause recurrent infections in individuals who already possess neutralizing antibodies against them (Whitley et al 1998).

The spectrum of pathology caused by HSV include infections of the skin and mucous membranes (eg, orolabial herpes and genital herpes), keratoconjunctivitis, encephalitis, and neonatal HSV infection (Whitley et al 1998). The most common types of HSV infections are genital herpes and orolabial herpes (Simpson and LysengWilliamson 2006). Orolabial herpes infections are usually caused by HSV type 1 
(HSV-1), while 70\% of first episode genital herpes cases are caused by HSV type 2 (HSV-2) (Chosidow et al 2001). However, overlap exists between the two types (Nadelman and Newcomer 2000), and HSV-1 is becoming an increasingly important causative agent of genital herpes in developed countries. More than $57 \%$ of the US population between the ages of 14 and 49 are HSV-1 seropositive (Xu et al 2006). Prevalence rises with increasing age in a roughly linear fashion, globally reaching 60-90\% in older adults (Smith and Robinson 2002). HSV-2, however, affects a smaller proportion of the population, with only $10 \%$ of 15 - to 29 -year-olds showing seropositivity. By age 60 , the prevalence increases to approximately $35 \%$ (Whitley et al 1998).

While HSV-1 is typically acquired through non-sexual contact in childhood and adolescence, HSV-2 is transmitted through sexual contact and is one of the most common sexually transmitted diseases in the world (Xu et al 2006). Primary HSV-2 infection often reveals itself as painful vesicles, pustules, and ulcerations in the anogenital area (Whitley et al 1998; Jungmann 2006). In males, the eruption presents as vesicular lesions on an erythematous base on the penis, while in females, lesions occur on the cervix and vulva. These lesions umbilicate, erode, and form a crust before healing completely (Nadelman and Newcomer 2000). The incubation period is 2-10 days (Jungmann 2006), and lesions may be present for approximately 3 weeks (Whitley et al 1998). Viral shedding can occur throughout this entire period. Neutralizing antibodies develop within 2-3 weeks (Nadelman and Newcomer 2000).

Primary genital herpes is often more severe in women, who have a higher likelihood of developing complications, especially aseptic meningitis. In addition to burning and paresthesias at the affected site, both men and women may also experience dysuria and systemic symptoms such as fever, malaise, and localized inguinal adenopathy (Whitley et al 1998; Nadelman and Newcomer 2000).

Approximately $50 \%$ of patients with genital herpes will experience at least one episode of recurrence in their lifetime (Nadelman and Newcomer 2000). Recurrent disease tends to be shorter in duration, lasting 8-10 days (vs 3 weeks), with a shorter period of viral shedding (2-5 days). Lesions are less numerous and less severe; very few (approximately 3-5) vesicles may appear on the male penis, and the female may only experience vulvar irritation. Generally, systemic symptoms do not occur during recurrent episodes. Frequency of recurrence correlates with the severity of primary infections; individuals who had more severe primary infections tend to have a greater number of recurrences. Recurrence is also more common in younger patients and individuals infected with HSV-2 compared with HSV-1 (Whitley et al 1998; Nadelman and Newcomer 2000).

While primary genital herpes infections are rarely unrecognized, primary orolabial infections are usually subclinical. When symptomatic, primary herpetic gingivostomatitis is the most common presentation, resulting in intraoral grouped vesicles that evolve into pustules and erosions at the site of inoculation, with accompanying regional lymphadenopathy, fever, headache, malaise, and myalgias (Wolff et al 2005). Like genital herpes, the virus that causes the primary infection travels to sensory ganglia and remains latent until opportunity for recurrence. Recurrent orolabial herpes, or "cold sores," affect roughly one third of those who harbor the virus, variably presenting with prodromal burning and itching followed by a painful eruption of grouped vesicles on an erythematous base that erode and crust (Spruance et al 2006).

Although herpetic lesions in immunocompetent patients may heal spontaneously within 10 days, both orolabial and genital herpes are usually treated medically to alleviate patient discomfort and anxiety. Patients with severe, recurrent disease may suffer significant quality of life impairment secondary to pain and disfigurement (Lorette et al 2006). Treatment facilitates healing, minimizing the duration of discomfort associated with the lesions. Meanwhile, untreated genital herpes has been shown to facilitate transmission of human immunodeficiency virus (HIV) infection (Freeman et al 2006). Additionally, patients with recurrent genital herpes may be asymptomatic, increasing their risk of unknowingly transmitting the disease to their partners from viral shedding. Further goals of treatment thus include reduction of viral shedding to limit transmission.

Currently, two treatment options are available to patients with recurrent genital herpes: episodic and suppressive therapy. Suppressive therapy involves daily oral antiviral agents to prevent future recurrences and is typically reserved for patients with frequent and/or severe outbreaks (Tyring et al 2006; Whitley et al 2006). Past studies have shown that $48 \%$ and $72 \%$ of patients on valacyclovir and famciclovir suppression, respectively, remain recurrence-free after one year, and that suppressive therapy can decrease the transmission of genital herpes (Diaz-Mitoma et al 1998; Reitano et al 1998; Corey et al 2004). Patients who are not sexually active or who do not wish to take daily medication may find episodic therapy a more suitable option. With this alternative, antiviral treatment is initiated at the onset of a recurrent outbreak to limit disease progression (Tyring et al 2006; Whitley et al 2006). 
In contrast to genital herpes, suppressive therapy is not common practice in the management of recurrent orolabial herpes. As only a few small-scale studies have explored this as a treatment option, episodic therapy continues to be the mainstay of treatment (Baker and Eisen 2003).

In order to limit viral replication and subsequent tissue damage, antiviral therapy must be initiated within the first 24 hours after prodromal or symptom onset, when viral concentrations are at their peak (Tyring et al 2006). Patient-initiated episodic therapy allows for the introduction of therapy within this narrow therapeutic window and confers more control over the disease to the patients. Additionally, patients derive greater benefit from this method as patient-initiated episodic therapy has been shown to reduce healing time to a greater extent than physician-initiated therapy (Reichman et al 1984; Chosidow et al 2001).

Recent clinical trials have suggested that a single-day high-dose antiviral regimen given within the first 24 hours of symptom onset may effectively speed healing of herpetic lesions. This practice harbors tremendous potential in improving patient convenience and decreasing the cost of a course of antiviral therapy.

\section{Antiviral pharmacology}

Currently, the only oral antiviral agents approved for treatment of herpes simplex virus infections are the nucleoside analogues acyclovir $\left(\right.$ Zovirax $\left.^{\circledR}\right)$, valacyclovir $\left(\right.$ Valtrex $\left.^{\circledR}\right)$, and famciclovir (Famvir ${ }^{\circledR}$ ) (Whitley et al 1998; Nadelman and Newcomer 2000; Jungmann 2006). Acyclovir was the first antiviral agent to be used in the treatment of herpes infections, traditionally as a 5-day course (Reichman et al 1984). However, the poor bioavailability of acyclovir (approximately $20 \%$ ) necessitated 3-5 times daily dosing and prompted the search for more suitable agents (Crumpacker 1996; Jensen et al 2004). Valacyclovir, the oral prodrug of acyclovir, has an improved bioavailability of approximately $55 \%$ and is also an effective treatment option (Reitano et al 1998; Tyring et al 1998; Leone et al 2002). Famciclovir, the oral prodrug of penciclovir, was found to have an even more favorable bioavailability (77\%) (Pue and Benet 1993; Tyring et al 1998), in addition to a longer intracellular half-life and greater affinity for viral thymidine kinase than acyclovir (Vere Hodge and Perkins 1989; Earnshaw et al 1992).

\section{Mechanism of action}

Activation of acyclovir and penciclovir is dependent on viral thymidine kinase found in cells infected with herpesvirus. Penciclovir is rapidly converted to penciclovir-monophosphate by thymidine kinase and then further phosphorylated to penciclovir-triphosphate by other cellular enzymes. Similarly, acyclovir is converted intracellularly to acyclovirtriphosphate, although at a much slower rate. This is due to the 100-fold higher affinity that thymidine kinase has for penciclovir compared to acyclovir, leading to the more efficient phosphorylation of penciclovir and higher intracellular concentrations of penciclovir-triphosphate versus acyclovirtriphosphate (Boyd et al 1987; Vere Hodge and Perkins 1989; Earnshaw et al 1992; Pue et al 1994; Crumpacker 1996).

Both penciclovir-triphosphate and acyclovir-triphosphate are analogues of the naturally occurring nucleoside deoxyguanosine (dGTP) and compete with dGTP as a substrate for viral DNA polymerase. Insertion of these analogues inhibits viral DNA chain elongation, preventing replication of the viral genome. Although both penciclovir-triphosphate and acyclovirtriphosphate act by interfering with DNA polymerase, there are some differences in their inhibitory mechanisms. Penciclovirtriphosphate functions as a short-chain terminator, allowing a small degree of further DNA chain elongation at the 3' hydroxyl group of its acyclic side chain. Acyclovir-triphosphate, however, is an obligate chain terminator, leading to cessation of DNA chain elongation after its incorporation. Additionally, DNA polymerase has a higher affinity for acyclovir-triphosphate than penciclovir-triphosphate. Despite this fact, in a study simulating physiologic concentration of nucleosides, penciclovir-triphosphate was found to more efficiently inhibit chain elongation by DNA polymerase than acyclovir-triphosphate (Reardon and Spector 1989; Vere Hodge and Cheng 1993; Crumpacker 1996; Bacon et al 2003).

Overall, studies have indicated that penciclovir and acyclovir have similar efficacy versus varicella zoster virus (VZV), HSV-1, and HSV-2. This is possible despite the decreased affinity of DNA polymerase for penciclovir-triphosphate versus acyclovir-triphosphate given the aforementioned increased affinity of thymidine kinase for penciclovir and the 100-fold increased intracellular concentrations of penciclovir-triphosphate (Larsson et al 1986; Earnshaw et al 1992; Vere Hodge and Cheng 1993; Bacon 1996; Crumpacker 1996).

Plaque reduction assays have demonstrated similar activities of penciclovir and acyclovir when the compounds are present continuously (Bacon 1996). However, upon withdrawal of the compounds, inhibition of virus replication was sustained for a greater time period with penciclovir than acyclovir. This sustained antiviral activity of penciclovir-triphosphate is due to increased stability of penciclovir-triphosphate, leading to prolonged intracellular 
concentrations and half-lives of penciclovir-triphosphate compared to acyclovir-triphosphate. In HSV-1 and HSV-2 infected cells, the half-life of famciclovir is 10 and 20 hours, respectively. For acyclovir, the half-lives are 0.7 and 1 hour (Boyd et al 1987; Bacon 1996; Crumpacker 1996).

\section{Selectivity}

After absorption, penciclovir and acyclovir are taken up into both infected and uninfected cells. However, phosphorylation is dependent on viral thymidine kinase and occurs selectively in infected cells, sparing host DNA synthesis (Boyd et al 1987; Vere Hodge and Perkins 1989). Cellular DNA polymerases have a lower affinity for the analogue triphosphates when compared with viral DNA polymerases. Therefore, at antiviral doses, minimal concentrations of penciclovir (approximately $0.04 \mu \mathrm{mol} / \mathrm{L}$ ) are isolated from uninfected cells and there is little inhibition of cellular DNA polymerases with no effect on human DNA concentrations (Earnshaw et al 1992; Vere Hodge and Cheng 1993; Ilsley et al 1995; Bacon 1996; Crumpacker 1996; Bacon et al 2003).

\section{Discussion}

\section{Treatment options for genital herpes}

Acyclovir was the first antiviral agent to be used in the treatment of genital herpes, and as such, is the most extensively studied of the antiviral agents currently available. This drug is available in topical, oral, and intravenous (IV) forms (Nadelman and Newcomer 2000). In the distant past, topical acyclovir was used to treat recurrent genital herpes, but its ineffectiveness has been known for quite some time. A randomized, placebo-controlled, double-blind study found that after application of topical acyclovir 6 times daily for 5 days, only the duration of virus shedding from lesions was reduced compared to placebo. Differences between time to lesion crusting, lesion healing, new lesion formation, and cessation of pain were non-significant (Reichman et al 1983).

Intravenous acyclovir remains the most effective form of treatment for a primary genital herpes infection, leading to significant reduction in time to cessation of viral shedding and pain. Time to lesion healing is also 6 days faster than with placebo alone ( 8 days vs 14 days). However, as IV administration of acyclovir requires hospitalization, this route is reserved for patients with life-threatening disease or systemic complications (Whitley et al 1998).

\section{Oral agents}

The current standard of therapy is oral medication. In addition to acyclovir, valacyclovir and famciclovir are the other oral agents approved for treatment of recurrent genital herpes. All three drugs have similar efficacy in the treatment of genital herpes and have been shown to expedite lesion healing time by approximately 1-2 days when compared with placebo (Sacks et al 1996; Tyring et al 1998; Chosidow et al 2001). Their differences are revealed in their pharmacokinetic properties, which dictate their dosing schedules. Acyclovir was traditionally dosed as $200 \mathrm{mg}$ five times daily for 5 days, mainly due to its aforementioned unfavorable pharmacokinetic profile (Reichman et al 1984). Such a regimen is extremely inconvenient and may contribute to poor patient compliance. Recently, however, shorter 2- to 3-day high-dose regimens of acyclovir and valacyclovir have also proven effective (Reitano et al 1998; Tyring et al 1998; Leone et al 2002; Wald et al 2002).

\section{Single-day treatment}

Based on the recent successes of high-dose acyclovir and valacyclovir regimens and the unique pharmacokinetic profile of famciclovir, an international, multicenter, randomized, double-blind, placebo-controlled trial was conducted to evaluate the efficacy of single-day famciclovir as a patient-initiated treatment for recurrent genital herpes in immunocompetent patients (Aoki et al 2006). Selection criteria stipulated that participants be healthy individuals with proven HSV-2 infection and at least 4 recurrences in the preceding 12 months. If on suppressive antiviral therapy at the time of enrollment, subjects must have had at least 4 recurrences in the 12 months prior to initiation of suppressive therapy and must have agreed to discontinue this therapy. 329 patients were randomized and given single-day famciclovir $1000 \mathrm{mg}$ twice daily $(\mathrm{n}=163)$ or matching placebo $(n=166)$ to take within 6 hours of the onset of prodromal symptoms or genital herpes lesions at their next recurrence. Overall, the patient demographics and baseline disease characteristics were well matched between the treatment and placebo arms.

Patients were followed daily in the clinic for the first 5 days and then every other day until all lesions had healed or day 14 was reached. The primary efficacy endpoint examined was the time to healing of all non-aborted lesions, with secondary endpoints of efficacy of famciclovir in inducing aborted lesions (defined as lesions that did not progress beyond the papule stage), time to resolution of pain and all other lesion-associated symptoms (defined as burning, tingling, itching, tenderness, and pain), and the safety/tolerability of high-dose famciclovir.

The results of this study indicated that patients treated with single-day, high-dose famciclovir experienced almost a 2-day improvement in lesion healing time (1.8 days, 
$\mathrm{p}<0.001$; Table 1) and a significant reduction in time to resolution of all symptoms ( 2.1 days, $p<0.001$ ) when compared to patients treated with placebo. Furthermore, treatment with famciclovir halted progression to a full genital herpes outbreak in nearly one in four patients; aborted lesions were observed in $23.3 \%$ of patients in the treatment arm compared to only $12.7 \%$ in the placebo arm $(p=0.003)$. Finally, highdose famciclovir was well-tolerated by study participants, with only mild to moderate adverse events, which seemed to be transient in nature and similarly occurred in the placebo group. The most common side effects reported were headaches and GI distress (diarrhea, nausea, and abdominal pain), with headaches occurring more frequently in the treatment group (13.5\% vs 5.4\%) (Aoki et al 2006).

\section{Comparison of therapies}

Although no head-to-head trials have compared single-day famciclovir to the currently prescribed 2- to 5-day antiviral regimens, it appears to produce similar or better reductions in time to lesion healing and resolution of symptoms (Table 1). Additionally, single-day famciclovir also prevents progression to a full genital herpes outbreak as effectively as, or even more effectively than, other antiviral regimens. This single-day treatment design introduces a high load of antiviral agent into the body during the time of maximal viral replication, preventing the tissue damage and breakdown, and reducing symptoms that typically occur in recurrent episodes of genital herpes. Furthermore, single-day therapy maximizes patient convenience and minimizes cost (Table 2). To date, famciclovir is the only antiviral agent proven effective as single-day therapy of genital herpes.

\section{Treatment options for orolabial herpes Topical agents}

The first antiviral agents used for the treatment of recurrent orolabial herpes were formulated in creams. Clinical trials have shown that topical antivirals speed healing and lessen pain, but require diligent application to affected areas. The first FDA-approved non-prescription topical medication for orolabial herpes was docosanol $10 \%$ cream $\left(\right.$ Abreva $\left.^{\circledR}\right)$, which demonstrated an 18-hour shorter median time to healing in treated patients compared to placebo (Sacks et al 2001). Penciclovir 1\% cream $\left(\right.$ Denavir $\left.^{\circledR}\right)$ and acyclovir 5\% cream $\left(\right.$ Zovirax $^{\circledR}$ ) are the two available prescription topical agents. Penciclovir 1\% cream has been shown in 3 randomized, double-blind, placebo-controlled trials to shorten median healing time of cold sores by $0.7-1$ day and pain duration by $0.6-0.8$ days compared to vehicle controls (Spruance

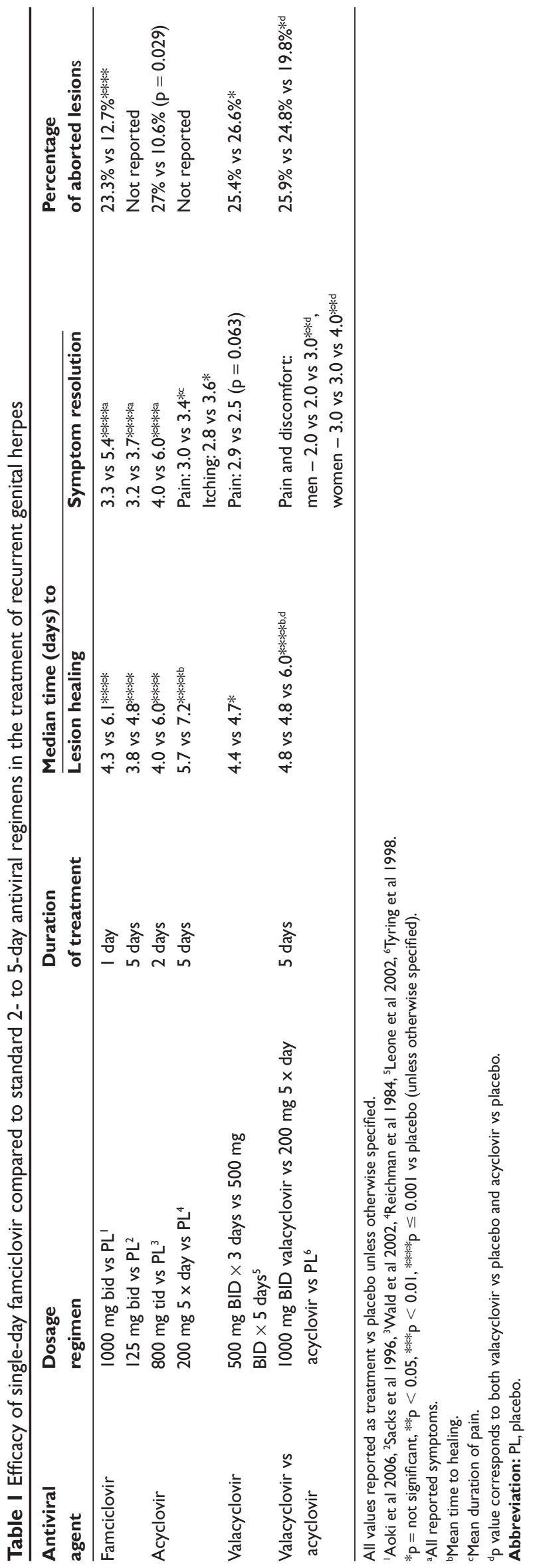


Table 2 Comparison of retail cost' of standard dosing of antiviral agents

\begin{tabular}{|c|c|c|}
\hline \multicolumn{3}{|c|}{ Retail cost of oral antivirals for orolabial herpes treatment } \\
\hline Drug & Dose & Price (\$) \\
\hline Famciclovir & $1500 \mathrm{mg}$ single dose (three $500 \mathrm{mg}$ tablets) & 28.09 \\
\hline Valacyclovir & $2 \mathrm{~g}$ bid $\times$ I day (four I g tablets) & 42.12 \\
\hline Acyclovir & $400 \mathrm{mg}$ tablets 5 times/day $\times 5$ days & 12.08 \\
\hline \multicolumn{3}{|c|}{ Retail cost of topical agents for orolabial herpes treatment } \\
\hline Drug & Dose & Price (\$) \\
\hline Penciclovir I\% cream & Every 2 hours, $1.5 \mathrm{~g}$ tube & 36.59 \\
\hline Acyclovir $5 \%$ cream & Five times/day, $2.0 \mathrm{~g}$ tube & 48.91 \\
\hline Docosanol $10 \%$ cream & Five times/day, $2.0 \mathrm{~g}$ tube & 14.99 \\
\hline \multicolumn{3}{|c|}{ Retail cost of oral antivirals for genital herpes treatment } \\
\hline Drug & Dose & Price (\$) \\
\hline \multirow[t]{2}{*}{ Famciclovir } & $125 \mathrm{mg}$ tablets bid $\times 5$ days & 47.47 \\
\hline & $1000 \mathrm{mg}$ bid $\mathrm{x}$ I day (four $500 \mathrm{mg}$ tablets) & 37.46 \\
\hline Valacyclovir & $500 \mathrm{mg}$ tablets bid $\times 3$ days & 33.60 \\
\hline Acyclovir & $800 \mathrm{mg}$ tablets tid $\times 2$ days & 7.99 \\
\hline
\end{tabular}

'Prices in US\$ cited from www.drugstore.com: Drug prices and information [accessed 22 July 2007].

et al 1997; Raborn et al 2002). Subjects in the penciclovir studies applied medication to the affected area within 1 hour of prodrome onset and continued to do so every 2 hours for 4 consecutive days. Acyclovir 5\% cream shortens the duration of episode by $0.5-0.6$ days and duration of pain by $0.3-0.4$ days when applied 5 times a day for 4 consecutive days (Spruance et al 2002). Interestingly, both topical acyclovir and penciclovir display therapeutic efficacy in early as well as late stage lesions (Spruance et al 2002).

\section{Oral agents}

Compared to topical treatments, oral agents offer the benefit of less frequent dosing and increased bioavailability. Acyclovir, famciclovir, and valacyclovir are the most commonly used oral antivirals in the treatment of orolabial herpes. Oral acyclovir has been shown in two clinical trials to have modest efficacy in decreasing healing time and duration of pain when given early in the prodromal stages of recurrent orolabial herpes. It has no effect on the development of secondary lesions. The current recommended dosing schedule is 400 mg 5 times a day for 5 days (Jensen et al 2004).

\section{Single-day treatments}

Famciclovir

Oral famciclovir given as a single $1500 \mathrm{mg}$ dose was FDA approved for the treatment of recurrent orolabial herpes in immunocompetent patients after the seminal work by Spruance and coworkers in 2006 (Spruance et al 2006). This randomized, double-blind, placebo-controlled study had patients self-initiating either a single dose of $1500 \mathrm{mg}$ famciclovir $(\mathrm{n}=227), 750 \mathrm{mg}$ famciclovir twice a day for a single day $(n=220)$, or placebo $(n=254)$, within 1 hour of prodromal symptoms. Subjects returned to the clinic within 24 hours for assessment. The primary efficacy endpoint was healing, defined by loss of crust and re-epithelialization, of the primary vesicular lesion. The secondary efficacy variables were time to healing of all vesicular lesions (primary and secondary), time to return to normal skin for all lesions (vesicular and aborted), and duration of pain.

Both famciclovir arms significantly decreased median time to healing of primary vesicular lesions vs. placebo by 1.8-2.2 days. All lesions healed a median of 2.1-2.5 days sooner compared to placebo. Healing time was not significantly different between the two treatment arms. Secondary lesions occurred less frequently in the famciclovir-treated patients ( $11 \%$ in treated vs $18 \%$ in placebo). Compared to placebo, the single-dose regimen significantly $(\mathrm{p}<0.001)$ lessened the duration of pain (1.7 vs 2.9 days) and time to return to normal skin (4.5 vs 7.0 days).

Incidence of headache and nausea in the treatment groups was similar to that of placebo. Headache was experienced by $9.7 \%, 7.3 \%$, and $6.7 \%$ of the single-dose ( $1500 \mathrm{mg})$, single-day (750 mg twice a day), and placebo groups, respectively. Nausea occurred in 2.2\%, 2.3\%, and 3.9\% (Spruance et al 2006).

Spruance's study demonstrated that a single $1500 \mathrm{mg}$ dose of famciclovir by mouth reduces healing time of cold sores by approximately 2 days and duration of pain by approximately 1 day compared to placebo (Table 3 ), offering a safe and effective alternative for patients on episodic therapy for recurrent orolabial herpes.

\section{Valacyclovir}

Rapidly absorbed and hydrolyzed into acyclovir and L-valine, valacyclovir has three to five times the bioavailability of 
traditional oral acyclovir (Ormrod and Goa 2000; Spruance et al 2003). Two large double-blind, randomized, placebocontrolled studies demonstrated that $2 \mathrm{~g}$ of valacyclovir twice a day for 1 day (single-day treatment) and $2 \mathrm{~g}$ of valacyclovir given twice a day for 1 day followed by $1 \mathrm{~g}$ twice a day for 1 day (2-day treatment) both significantly shortened the time to healing of cold sore lesions and duration of pain compared to placebo. Patients initiated treatment at the first sign of prodromal symptoms and appeared in clinic within 24 hours for evaluation. The primary efficacy measure in Study 1 was the duration of the episode, measured from the start of treatment until healing, as defined by loss of crust on vesicular lesions and return to normal skin on non-vesicular lesions. The primary efficacy measure in Study 2 was the proportion of subjects in which cold sore development was prevented.

In the first study, the single-day $(\mathrm{n}=311)$ and two-day $(n=299)$ treatment groups experienced shortened duration of episode by a mean of $1.1(\mathrm{p}<0.001)$ and 0.7 days $(\mathrm{p}=0.008)$, respectively, compared to placebo $(n=292)$. The second study had similar results, with both treatment arms (singleday $n=298$, 2-day $n=339$ ) experiencing a shortened mean disease duration of 1.0 and 0.8 days compared to placebo $(\mathrm{n}=317)$. There was no added benefit in treating for 2 days vs 1 day. Development of new lesions was decreased in the treated groups, but not to a statistically significant amount compared to placebo. The only adverse event that occurred with a greater frequency in the treated subjects was headache: placebo (4\%-5\%), single-day (9\%-10\%), 2-day (9\%) (Spruance et al 2003).

These two studies show that single-day high dose ( $2 \mathrm{~g}$ bid $)$ course of valacyclovir safely and effectively reduces healing time of cold sores by an average of 1 day compared to placebo. There is no additional benefit in treating for 2 days.

\section{Comparison of therapies}

While clinical trials show that topical agents are efficacious, patients in the real world are unlikely to comply with the frequent dosing schedule required for optimal results, making oral antiviral agents the most convenient option for patientinitiated episodic therapy. Although no head-to-head comparisons of orolabial herpes therapies exist, oral famciclovir and valacyclovir appear to offer similar reductions in duration of lesions and associated pain (Table 3). Given the similar efficacy of these agents, perhaps prescribing practices should also be guided by a patient's overall financial circumstance, and accordingly we have included a cost-list of commonly used agents (Table 2).

\section{Conclusion}

Mucocutaneous infections caused by herpes simplex viruses in immunocompetent patients are usually self-limiting, although their impact on patient quality of life can range from minor annoyance to severe disability. There are several well-recognized treatment strategies in existence, but single-day oral antiviral therapy for episodic treatment of recurrences is a new practice that has only recently been validated by several well-designed, large-scale clinical trials. While there are no studies that directly compare the efficacy of different oral antivirals, available safety and efficacy data support famciclovir $1500 \mathrm{mg}$ once a day or valacyclovir $2 \mathrm{~g}$ bid for 1 day in recurrent orolabial herpes as suitable first-line therapies for maximizing patient convenience and

Table 3 Efficacy of single-day famciclovir compared to single-day valacyclovir in the treatment of recurrent orolabial herpes

\begin{tabular}{|c|c|c|c|c|c|}
\hline Antiviral agent & Study design & $\begin{array}{l}\text { Dosing } \\
\text { regimen }\end{array}$ & $\begin{array}{l}\text { Time to loss of crust } \\
\text { (median days) }\end{array}$ & $\begin{array}{l}\text { Time to loss of crust } \\
\text { (mean days) }\end{array}$ & $\begin{array}{l}\text { Duration of pain } \\
\text { (median days) }\end{array}$ \\
\hline \multirow[t]{4}{*}{ Famciclovir } & $R, D B, P C$ & Patient-initiated & & & \\
\hline & $\mathrm{n}=70 \mathrm{I}$ & $1500 \mathrm{mg}$ single dose & $4.4^{* * *}$ & NA & $1.7(p<0.001)$ \\
\hline & & $750 \mathrm{mg}$ bid for I day & $4.0 * * *$ & NA & $2.1(p=0.054)$ \\
\hline & & placebo & 6.0 & NA & 2.9 \\
\hline Valacyclovir $^{2}$ & $\mathrm{R}, \mathrm{DB}, \mathrm{PC}$ & Patient-initiated & & & \\
\hline \multirow[t]{4}{*}{ Study I } & $\mathrm{n}=902$ & $2 \mathrm{~g}$ bid for I day & $4.0 * * *$ & $5.0(p<0.001)$ & $1.2(p=0.009)$ \\
\hline & & $2 \mathrm{~g}$ bid for day $\mathrm{I}$ & $4.5(p=0.009)$ & $5.3(p=0.008)$ & $1.3(p=0.008)$ \\
\hline & & I g bid for day 2 (2-day) & & & \\
\hline & & placebo & 5.0 & 6.1 & 1.8 \\
\hline \multirow[t]{4}{*}{ Study 2} & $\mathrm{n}=954$ & $2 \mathrm{~g}$ bid for one day & $5.0 * * *$ & 5.3 & $1.5(p<0.001)$ \\
\hline & & $2 \mathrm{~g}$ bid for day $\mathrm{I}$ & $5.0 * * *$ & 5.5 & $1.5(p=0.003)$ \\
\hline & & I g bid for day 2 (2-day) & & & \\
\hline & & placebo & 5.5 & 6.3 & 2.2 \\
\hline
\end{tabular}

'Spruance et al 2006, ${ }^{2}$ Spruance et al 2003.

${ }^{*} p<0.05$, ** $p<0.01$, *** $\mathrm{p} \leq 0.00$ I vs placebo.

Abbreviations: DB, double-blind; PC, placebo-controlled; R, randomized.

Therapeutics and Clinical Risk Management 2008:4(2) 
minimizing cost. Likewise in recurrent genital herpes, famciclovir $1000 \mathrm{mg}$ bid for 1 day appears to produce similar or better reductions in time to lesion healing and resolution of symptoms than the currently prescribed 2- to 5-day antiviral regimens. Additionally, single-day famciclovir is effective at preventing progression to a full genital herpes outbreak and is well-tolerated. These characteristics indicate single-day famciclovir to be appropriate as a first-line therapy.

Single-day treatment for recurrent orolabial and genital herpes introduces a high load of antiviral agent into the body during the time of maximal viral replication, limiting progression of disease. With single-day therapy, patients experience faster healing time, shorter duration of symptoms, while simultaneously regaining control over their disease through patient-initiated therapy.

\section{Disclosures}

Dr. Tyring has conducted research and been a consultant for Novartis and GlaxoSmithKline. He has also received honoraria and grants from the same.

\section{References}

Aoki FY, Tyring S, Diaz-Mitoma F, et al. 2006. Single-day, patient-iniated famciclovir therapy for recurrent genital herpes: a randomized, doubleblind, placebo-controlled trial. Clin Infect Dis, 24:8-13.

Bacon TH. 1996. Famciclovir, from the bench to the patient - a comprehensive review of preclinical data. Int J Antimicrob Agents, 7:119-34.

Bacon TH, Levin MJ, Leary JJ, et al. 2003. Herpes simplex virus resistance to acyclovir and penciclovir after two decades of antiviral therapy. Clin Microb Rev, 16:114-28.

Baker D, Eisen D. 2003. Valacyclovir for prevention of recurrent herpes labialis: 2 double-blind, placebo-controlled studies. Cutis, 71:239-42.

Boyd MR, Bacon TH, Sutton D, et al. 1987. Antiherpesvirus activity of 9-(4-hydroxy-3-hydroxy-methylbut-1-yl)guanine (BRL 39123) in cell culture. Antimicrob Agents Chemother, 31:1238-42.

Chosidow O, Drouault Y, Leconte-Veyriac F, et al. 2001. Famciclovir vs. aciclovir in immunocompetent patients with recurrent genital herpes infections: a parallel-groups randomized, double-blind clinical trial. Br J Dermatol, 144:818-824.

Corey L, Wald A, Patel R, et al. 2004. Once-daily valacyclovir to reduce the risk of transmission of genital herpes. N Engl J Med, 350:11-20.

Crumpacker C. 1996. The pharmacological profile of famciclovir. Semin Dermatol, 15:14-26.

Diaz-Mitoma F, Sibbald RG, Shafran SD, et al. 1998. Oral famciclovir for the suppression of recurrent genital herpes: a randomized controlled trial. Collaborative Famciclovir Genital Herpes Research Group. JAMA, 280:887-92.

Earnshaw DL, Bacon TH, Darlison SJ, et al. 1992. Mode of antiviral action of penciclovir in MRC-5 cells infected with herpes simplex virus type 1 (HSV-1), HSV-2, and varicella-zoster virus. Antimicrob Agents Chemother, 36:2747-57.

Freeman EE, Weiss HA, Glynn JR, et al. 2006. Herpes simplex virus 2 infection increases HIV acquisition in men and women: systematic review and meta-analysis of longitudinal studies. Aids, 20:73-83.

Ilsley DD, Lee S-H, Miller WH, et al. 1995. Acyclic guanosine analogs inhibit DNA polymerases $\alpha, \delta$, and $\varepsilon$ with very different potencies and have unique mechanisms of action. Biochemistry, 34:2504-10.

Jensen LA, Hoehns JD, Squires CL. 2004. Oral antivirals for the acute treatment of recurrent herpes labialis. Ann Pharmacother, 38:705-709.
Jungmann E. 2006. Genital herpes. Clin Evid, Aug:1-13.

Larsson A, Stenberg K, Ericson A-C, et al. 1986. Mode of action, toxicity, pharmacokinetics, and efficacy of some new antiherpesvirus guanosine analogs related to buciclovir. Antimicrob Agents Chemother, 30:598-605.

Leone PA, Trottier S, Miller JM. 2002. Valacyclovir for episodic treatment of genital herpes: a shorter 3-day treatment course compared with 5-day treatment. Clin Infect Dis, 34:958-62.

Lorette G, Crochard A, Mimaud V, et al. 2006. A survey on the prevalence of orofacial herpes in France: the INSTANT Study. J Am Acad Dermatol, 55:225-,32.

Nadelman CM and Newcomer VD. 2000. Herpes simplex virus infections: new treatment approaches make early diagnosis even more important. Postgrad Med, 107:189-200.

Ormrod D, Goa K. 2000. Valaciclovir: a review of its use in the management of herpes zoster. Drugs, 59:1317-40.

Pue MA, Benet LZ. 1993. Pharmacokinetics of famciclovir in man. Antivir Chem Chemother, 4:47-55.

Pue MA, Pratt SK, Fairless AJ, et al. 1994. Linear pharmacokinetics of penciclovir following administration of single oral doses of famciclovir 125, 250, 500 and $750 \mathrm{mg}$ to healthy volunteers. J Antimicrob Chemother, 33:119-27.

Raborn GW, Martel AY, Lassonde M, et al. 2002. Effective treatment of herpes simplex labialis with penciclovir cream: combined results of two trials. J Am Dent Assoc, 133:303-9.

Reardon JE, Spector T. 1989. Herpes simplex virus type 1 DNA polymerase: mechanism of inhibition by acyclovir triphosphate. $J$ Biol Chem, 264:7405-11.

Reichman RC, Badger GJ, Guinan ME, et al. 1983. Topically administered acyclovir in the treatment of recurrent herpes simplex genitalis: a controlled trial. J Infect Dis, 147:336-40.

Reichman RC, Badger GJ, Mertz GJ, et al. 1984. Treatment of recurrent genital herpes simplex infections with oral acyclovir: a controlled trial. JAMA, 251:2103-7.

Reitano M, Tyring S, Lang W, et al. 1998. Valaciclovir for the suppression of recurrent genital herpes simplex virus infection: a large-scale dose range-finding study. International Valaciclovir HSV Study Group. J Infect Dis, 178:603-10.

Sacks SL, Aoki FY, Diaz-Mitoma F, et al. 1996. Patient-initiated, twicedaily oral famciclovir for early recurrent genital herpes: a randomized, double-blind multicenter trial. JAMA, 276:44-9.

Sacks SL, Thisted RA, Jones TM, et al. 2001. Clinical efficacy of topical docosanol $10 \%$ cream for herpes simplex labialis: A multicenter, randomized, placebo-controlled trial. J Am Acad Dermatol, 45:222-30.

Simpson D and Lyseng-Williamson KA. 2006. Famciclovir: a review of its use in herpes zoster and genital and orolabial herpes. Drugs, 66:2397-416.

Smith JS and Robinson NJ. 2002. Age-specific prevalence of infection with herpes simplex virus types 2 and 1: a global review. J Infect Dis, 186:S3-S28.

Spruance SL, Bodsworth N, Resnick H, et al. 2006. Single-dose, patientinitiated famciclovir: A randomized, double-blind, placebo-controlled trial for episodic treatment of herpes labialis. J Am Acad Dermatol, 55:47-53.

Spruance SL, Jones TM, M.M. B, et al. 2003. High-dose, short-duration, early valacyclovir therapy for episodic treatment of cold sores: results of two randomized, placebo-controlled, multicenter studies. Antimicrob Agents Chemother, 43:1072-80.

Spruance SL, Nett R, Marbury T, et al. 2002. Acyclovir Cream for Treatment of Herpes Simplex Labialis: Results of Two Randomized, Double-Blind, Vehicle-Controlled, Multicenter Clinical Trials. Antimicrob Agents Chemother, 46:2238-43.

Spruance SL, Rea TL, Thoming C, et al. 1997. Penciclovir Cream for the Treatment of Herpes Simplex Labialis: A Randomized, Multicenter, Double-blind, Placebo-Controlled Trial. JAMA, 277:1374-479.

Tyring S, Berger T, Yen-Moore A, et al. 2006. Single-day therapy for recurrent genital herpes. Am J Clin Dermatol, 7:209-11. 
Tyring SK, Douglas JM Jr, Corey L, et al. 1998. A randomized, placebo-controlled comparison of oral valacyclovir and acyclovir in immunocompetent patients with recurrent genital herpes infections. Arch Dermatol, 134:185-91.

Vere Hodge RA and Cheng Y-C. 1993. The mode of action of penciclovir. Antivir Chem Chemother, 4:13-24.

Vere Hodge RA, Perkins RM. 1989. Mode of action of 9-(4-hydroxy-3hydroxymethylbut-1-yl)guanine (BRL 39123) against herpes simplex virus in MRC-5 cells. Antimicrob Agents Chemother, 33:223-9.

Wald A, Carrell D, Remington M, et al. 2002. Two-day regimen of acyclovir for treatment of recurrent genital herpes simplex virus type 2 infection. Clini Infect Dis, 34:944-8.
Whitley R, Diaz-Mitoma F, Hamed K. 2006. Single-day famciclovir therapy for recurrent genital herpes. Curr Medl Res Opin, 22:1307-10.

Whitley RJ, Kimberlin DW and Roizman B. 1998. Herpes simplex viruses. Clin Infect Dis, 26:541-53.

Wolff K, Johnson RA, Suurmond D. 2005. Nongenital herpes simplex virus infection. Fitzpatrick's color atlas and synopsis of clinical dermatology. McGraw-Hill. pp. 802-5.

Xu F, Sternberg MR, Kottiri BJ, et al. 2006. Trends in herpes simplex virus type 1 and type 2 seroprevalence in the United States. JAMA, 296:964-73. 
